\title{
Efficiency of p16 and Ki-67 immunostaining for detecting premalignant cervical lesions in high risk population
}

\author{
Bugra Sahin $^{1}$, Dagistan Tolga Arioz ${ }^{1}$, Mine Kanat-Pektas ${ }^{1 *}$, \\ Cigdem Ozdemir², Gizem Cura'
}

\begin{abstract}
${ }^{1}$ Department of Gynecological Oncology, ${ }^{2}$ Department of Pathology, Afyonkarahisar Health Sciences University Hospital, Afyonkarahisar, Turkey
\end{abstract}

Received: 12 May 2021

Revised: 06 June 2021

Accepted: 07 June 2021

\section{*Correspondence:}

Dr. Mine Kanat-Pektas,

E-mail: minekanat@hotmail.com

Copyright: () the author(s), publisher and licensee Medip Academy. This is an open-access article distributed under the terms of the Creative Commons Attribution Non-Commercial License, which permits unrestricted non-commercial use, distribution, and reproduction in any medium, provided the original work is properly cited.

\begin{abstract}
Background: This study aimed to evaluate the efficiency of immunostaining with p16 and Ki-67 in cervical cytology specimens for the detection of cervical intraepithelial neoplasia (CIN) in a high risk population.

Methods: This was a prospective review of 287 women who underwent pap smear, human papilloma virus (HPV) testing and colposcopy examination, respectively. There were cervical smear abnormalities in 108 women (37.6\%) and 141 patients (49.1\%) tested positive for HPV. Cervical biopsy revealed normal cervix in 28 patients $(9.75 \%)$, cervicitis in 48 patients $(16.72 \%)$, CIN1 in 178 patients $(62.02 \%)$, CIN2 in 26 patients $(9.05 \%)$ and CIN3 in 7 patients $(2.43 \%)$. Results: Positive staining for p16 had a sensitivity of $78.2 \%$ and a specificity of $97.4 \%$ while positive staining for Ki67 had a sensitivity of $80.6 \%$ and a specificity of $57.9 \%$ for distinguishing CIN lesions in cervical cytology specimens $(\mathrm{p}=0.001$ for both). Concurrent positive staining for p16 and Ki67 in cervical cytology specimens had a sensitivity of $80.6 \%$ and a specificity of $97.4 \%$ for CIN lesions $(\mathrm{p}=0.001)$. Positive staining for $\mathrm{p} 16$ had a sensitivity of $94 \%$ and a specificity of $90.6 \%$ whereas positive staining for $\mathrm{Ki} 67$ had a sensitivity of $97 \%$ and a specificity of $33 \%$ for differentiating CIN lesions in colposcopic biopsy specimens ( $\mathrm{p}=0.001$ for both). Concurrent positive staining for $\mathrm{p} 16$ and Ki67 in colposcopic biopsy specimens had a sensitivity of $91 \%$ and a specificity of $94 \%$ for CIN lesions ( $\mathrm{p}=0.001$ ). Conclusions: p16/Ki-67 immunostaining applied on cervical cytology specimens can screen CIN lesions with high sensitivity and specificity in a low risk population.
\end{abstract}

Keywords: Cervical intraepithelial neoplasia, Colposcopy, Human papilloma virus, Immunohistochemistry, Pap smear

\section{INTRODUCTION}

Cervical cancer is the fourth most frequent female cancer which accounts for $6.6 \%$ of all cancers emerging in women. ${ }^{1}$ The most crucial step in the pathogenesis of cervical cancer is the integration of HPV DNA sequences into the host genome. ${ }^{2}$ This integration occurs in accordance with the loss of E2 tumor suppressor gene which regulates the expression of E6 and E7 oncogenes. ${ }^{3}$
These high risk oncogenes bind and inactivate the tumor suppressor proteins $\mathrm{p} 53$ and $\mathrm{Rb}$ respectively, leading to abnormal cell proliferation. ${ }^{4}$ In other words, the activation of E6 and E7 oncogenes enhances the transformation of HPV infections into carcinogenesis. ${ }^{2-4}$

CIN are the precursor lesions of invasive cervical carcinoma. These lesions are classified into mild (grade 1, CIN1), moderate (grade 2, CIN2) and severe (grade 3, CIN3) subtypes on the basis of the extent of epithelial 
involvement. ${ }^{5}$ Although CIN1 is usually not precancerous and does not require treatment, CIN $2 / 3$ has a risk of $10 \%$ to $40 \%$ for progression into cervical cancer. . $^{6,7}$

The cyclin-dependent kinase inhibitor which regulates the proliferation in G1-S phase of cell cycle is p16. This protein impairs cell proliferation through a reciprocal relationship with $\mathrm{Rb}$ protein. That is, the expression of $\mathrm{p} 16$ is increased as $\mathrm{Rb}$ is inactivated by HPV infection. ${ }^{8,9} \mathrm{Ki}-$ 67 is a nuclear and nucleolar protein which is expressed only in active G1, S, G2 and M phases of cell cycle. It is well known that the expression of Ki-67 directly correlates with cell proliferation. ${ }^{10}$ Since HPV triggers epithelial proliferation, the increase in $\mathrm{Ki}-67$ expression may indicate HPV infection. ${ }^{11,12}$ Therefore, it has been hypothesized that p16 and Ki-67 can be used to specify persistent infections with high risk HPV types. ${ }^{12}$

This study aimed to evaluate the efficiency of immunohistochemical staining with p16 and Ki-67 for the detection of premalignant lesions in cervical cytology specimens of a low risk population.

\section{METHODS}

This is a prospective review of 287 women with high risk for cervical cancer who were consecutively admitted to the department of gynecological oncology at Afyonkarahisar health sciences university hospital between January 2016 and June 2017. High risk for cervical cancer referred to having a cervix with abnormal appearance $(\mathrm{N}=77)$, smoking $(\mathrm{N}=72)$, having a history of oral contraceptive use $(\mathrm{N}=53)$, having a history of sexually transmitted diseases $(\mathrm{N}=44)$, having multiple sexual partners $(\mathrm{N}=26)$ and grand multiparity $(\mathrm{N}=15)$. Each participant underwent pap smear, HPV testing and colposcopy examination respectively at the study center. This study was approved by institutional review board and ethical committee of Afyon health sciences university hospital (grant no: 2018/E.19673). All patients were informed about the study design and their written consent was obtained.

The patients with pregnancy, patients with immunedeficiency, patients who underwent cervical surgery and the patients who had cervical cancer were excluded. Data related with age, marital age, previous pregnancies, smoking and oral contraceptive use were recorded.

\section{Liquid based cytology and HPV testing}

Cytological abnormalities were designated using liquid based technology (thin prep 2000 processor, Cytyc Corporation, Marlborough, MA, USA). According to the manufacturer's instructions, thin layer slides were prepared and cytological abnormalities were defined based on the Bethesda reporting system criteria. ${ }^{13}$ Cervical smear abnormalities consisted of atypical squamous cell of undetermined significance (ASCUS) $(\mathrm{N}=69)$, atypical squamous cells that cannot exclude a high-grade squamous intraepithelial lesion $(\mathrm{N}=15)$ and low grade squamous intraepithelial lesion (LSIL) $(\mathrm{N}=24)$.

A total of 141 patients $(49.1 \%)$ tested positive for high risk HPV DNA which was detected by hybrid capture 2 assay (HC2, Digene, Gaithersburg, MD, USA). In case the number of RLU/CO was equal to or greater than 1.0, HPV DNA was considered to be positive.

\section{Colposcopy examination}

Colposcopy was performed by means of Olympus Evis Exera II CV-180 equipment (Olympus, Barcelona, Spain) after preparing the cervix with $5 \%$ acetic acid. A colposcopically directed biopsy was obtained whenever an abnormal area was visualized. If transformation was zone was completely visualized and there were no abnormal colposcopy findings, a random biopsy was taken from the transformation zone.

\section{Histopathological examination}

Colposcopic biopsy specimens were examined independently by two experienced pathologists who did not know the results of cytological assessment and immunohistochemical staining. Whenever the pathologists yielded different diagnoses, cervical biopsy specimens were re-examined for a consensus result. The final histopathological diagnoses were made as follows, normal cervical tissue in 28 patients $(9.75 \%)$, cervicitis in 48 patients (16.75\%), CIN1 in 178 patients $(62.02 \%), \mathrm{CIN} 2$ in 26 patients $(9.05 \%)$ and CIN3 in 7 patients $(2.43 \%)$.

\section{Immnuhistochemical staining}

Liquid based cervical cytology specimens were all subjected to p16 and Ki-67 immunohistochemistry using a T2000 slide processor (Hologic, Bedford, MA, USA) according to the manufacturer's instructions. If at least one cervical epithelial cell was colored both with a brown cytoplasmic stain (p16) and a red nuclear stain (Ki67), this case was considered as positive for p16/Ki67 immunocytochemistry. ${ }^{14}$

Immunohistochemical staining was also performed on 1.5 $\mu \mathrm{m}$ sections acquired from formaldehyde fixed and paraffin embedded cervical tissues. According to the manufacturer's instructions, rabbit monoclonal antibody clone R19-D (DB Biotech, Kosice, Slovakia) and rabbit monoclonal antibody clone SP6 (Thermo Fisher Scientific, MA, USA) were used for immunohistochemical staining with p16 and Ki-67 respectively.

\section{Statistical analysis}

Collected data were analyzed by statistical package for social sciences version 22.0 (SPSS IBM, Armonk, NY, USA). Continuous variables were expressed as mean \pm standard deviation (range: minimum-maximum) while categorical variables were denoted as numbers or 
percentages where appropriate. Diagnostic sensitivity and specificity were computed by Chi square test and the results were specified within $95 \%$ confidence intervals. Two tailed $\mathrm{p}$ values $<0.01$ were accepted to be statistically significant.

\section{RESULTS}

Table 1 shows the demographic and clinical characteristics of the participants. When compared to the patients with normal cervix, the patients with CIN2 had significantly older age and marital age $(p=0.001$ for both). The frequency of smoking was significantly higher in the patients with CIN2 than the patients with cervicitis $(p=0.001)$. Grand multiparity was significantly more frequent in patients with CIN3 than patients with normal cervix $(\mathrm{p}=0.001)$.

Table 2 demonstrates the cervical cytology, HPV DNA and immunostaining results with respect to final histopathological diagnoses. When compared to the patients with normal cervix, cervical cytology abnormality and HPV DNA positivity were significantly more frequent in patients with CIN2 and CIN3 ( $\mathrm{p}=0.001$ for both).
Similarly, p16 and Ki-67 positivity in cervical cytology specimens and p16 and Ki-67 positivity in colposcopic biopsy specimens were significantly more frequent in patients with CIN2 and CIN3 than the patients with normal cervix $(\mathrm{p}=0.001$ for all).

Table 3 displays that positive staining for p16 in cervical cytology specimens had a sensitivity of $78.2 \%$ and a specificity of $97.4 \%$ while positive staining for Ki-67 in cervical cytology specimens had a sensitivity of $80.6 \%$ and a specificity of $57.9 \%$ for distinguishing CIN lesions ( $\mathrm{p}=0.001$ for both). Positive staining for both $\mathrm{p} 16$ and $\mathrm{Ki}-$ 67 in cervical cytology specimens had a sensitivity of $80.6 \%$ and a specificity of $97.4 \%$ for CIN lesions $(\mathrm{p}=0.001)$.

Table 4 indicates that positive staining for p16 in colposcopic biopsy specimens had a sensitivity of $94 \%$ and a specificity of $90.6 \%$ whereas positive staining for Ki-67 had a sensitivity of $97 \%$ and a specificity of $33 \%$ for differentiating CIN lesions ( $\mathrm{p}=0.001$ for both). Positive staining for both p16 and Ki-67 in colposcopic biopsy specimens had a sensitivity of $91 \%$ and a specificity of 94\% for CIN lesions ( $\mathrm{p}=0.001)$.

Table 1: Demographic and clinical characteristics of the participants.

\begin{tabular}{|lllllll|}
\hline Demographics & $\begin{array}{l}\text { Normal } \\
(\mathbf{N}=\mathbf{2 8})\end{array}$ & $\begin{array}{l}\text { Cervicitis } \\
(\mathbf{N}=48)\end{array}$ & $\begin{array}{l}\text { CIN1 } \\
(\mathbf{N}=178)\end{array}$ & $\begin{array}{l}\text { CIN2 } \\
(\mathbf{N}=26)\end{array}$ & $\begin{array}{l}\text { CIN 3 } \\
(\mathbf{N}=7)\end{array}$ & $\begin{array}{l}\text { P } \\
\text { value }\end{array}$ \\
\hline Age (in years) & $42.5 \pm 1.6$ & $44.3 \pm 2.1$ & $44.8 \pm 2.5$ & $47.4 \pm 3.7$ & $49.5 \pm 3.9$ & $0.001 *$ \\
\hline Oral contraceptive use (\%) & $4(14.3)$ & $3(6.3)$ & $41(23.0)$ & $4(15.4)$ & $1(14.3)$ & 0.149 \\
\hline Smoking (\%) & $11(39.3)$ & $8(16.7)$ & $35(19.7)$ & $14(53.8)$ & $2(28.6)$ & $0.001 *$ \\
\hline Marital age (in years) & $24.5 \pm 3.7$ & $23.7 \pm 2.9$ & $21.9 \pm 1.5$ & $20.5 \pm 2.8$ & $19.7 \pm 1.6$ & $0.001 *$ \\
\hline Nulliparity (\%) & $3(10.7)$ & $4(8.3)$ & $0(0.0)$ & $3(11.5)$ & $0(0.0)$ & $0.002 *$ \\
\hline Grand multiparity $(\%)$ & $0(0.0)$ & $0(0.0)$ & $6(3.4)$ & $5(19.2)$ & $4(57.1)$ & $0.001 *$ \\
\hline
\end{tabular}

$* \mathrm{p}<0.05$ was accepted to be statistically significant.

Table 2: Cervical cytology, HPV DNA and immunostaining results.

\begin{tabular}{|lllllll|}
\hline Results & $\begin{array}{l}\text { Normal } \\
(\mathbf{N}=\mathbf{2 8})(\%)\end{array}$ & $\begin{array}{l}\text { Cervicitis } \\
(\mathbf{N}=48)(\%)\end{array}$ & $\begin{array}{l}\text { CIN1 } \\
(\mathbf{N}=178)(\%)\end{array}$ & $\begin{array}{l}\text { CIN2 } \\
(\mathbf{N}=26)(\%)\end{array}$ & $\begin{array}{l}\text { CIN3 } \\
(\mathbf{N}=7)(\%)\end{array}$ & $\begin{array}{l}\text { P } \\
\text { value }\end{array}$ \\
\hline Abnormal cervical cytology & $3(10.7)$ & $11(22.9)$ & $94(52.8)$ & $26(100.0)$ & $7(100.0)$ & $0.001^{*}$ \\
\hline HPV DNA positivity & $0(0.0)$ & $0(0.0)$ & $75(42.1)$ & $26(100.0)$ & $7(100.0)$ & $0.001^{*}$ \\
\hline Cervical cytology & & & & & \\
\hline p16 positivity & $1(3.5)$ & $2(4.2)$ & $102(57.3)$ & $22(84.6)$ & $7(100.0)$ & $0.001^{*}$ \\
\hline Ki-67 positivity & $2(7.1)$ & $4(8.3)$ & $135(75.8)$ & $24(92.3)$ & $7(100.0)$ & $0.001^{*}$ \\
\hline Colposcopic biopsy & & & & & \\
\hline p16 positivity & $0(0.0)$ & $1(2.1)$ & $112(62.9)$ & $24(92.3)$ & $7(100.0)$ & $0.001^{*}$ \\
\hline Ki-67 positivity & $1(3.5)$ & $2(4.2)$ & $138(77.5)$ & $25(96.1)$ & $7(100.0)$ & $0.001^{*}$ \\
\hline
\end{tabular}

$* \mathrm{p}<0.05$ was accepted to be statistically significant.

Table 3: Immunostaining in cervical cytology specimens for intraepithelial lesions.

\begin{tabular}{|llll|}
\hline Specimens & p16 positivity & Ki-67 positivity & \multicolumn{1}{c|}{$\begin{array}{l}\text { Concurrent } \\
\text { p16 and Ki-67 } \\
\text { positivity }\end{array}$} \\
\hline Sensitivity (\%) & $78.2(71.4-81.5)$ & $80.6(74.6-85.7)$ & $80.6(74.6-85.7)$ \\
\hline Specificity (\%) & $97.4(90.8-99.7)$ & $57.9(46.0-69.1)$ & $97.4(90.8-99.7)$ \\
\hline Positive predictive value (\%) & $96.4(86.9-99.1)$ & $84.2(80.2-87.5)$ & $95.6(84.2-98.9)$ \\
\hline
\end{tabular}




\begin{tabular}{|llll|}
\hline Specimens & p16 positivity & Ki-67 positivity & \multicolumn{1}{c|}{$\begin{array}{l}\text { Concurrent } \\
\text { p16 and Ki-67 } \\
\text { positivity }\end{array}$} \\
\hline $\begin{array}{l}\text { Negative predictive values } \\
(\%)\end{array}$ & $31.9(30.1-33.8)$ & $51.8(43.4-60.0)$ & $30.6(29.0-32.3)$ \\
\hline Positive likelihood ratio & $9.55(2.39-38.24)$ & $1.91(1.46-2.51)$ & $7.74(1.92-31.18)$ \\
\hline Negative likelihood ratio & $0.77(0.71-0.84)$ & $0.34(0.24-0.48)$ & $0.82(0.76-0.89)$ \\
\hline P value & $0.001^{*}$ & $0.001 *$ & $0.001^{*}$ \\
\hline
\end{tabular}

$* \mathrm{p}<0.05$ was accepted to be statistically significant.

Table 4: Immunostaining in colposcopic biopsy specimens for cervical intraepithelial lesions.

\begin{tabular}{|llll|}
\hline Specimens & p16 positivity & Ki-67 positivity & $\begin{array}{l}\text { Concurrent } \\
\text { p16 and Ki67 } \\
\text { positivity }\end{array}$ \\
\hline Sensitivity (\%) & $93.9(79.8-99.3)$ & $97.0(84.2-99.9)$ & $90.9(75.7-98.1)$ \\
\hline Specificity (\%) & $90.6(86.3-93.9)$ & $33.1(27.3-39.2)$ & $94.1(90.5-96.7)$ \\
\hline Positive predictive value (\%) & $56.4(46.6-65.6)$ & $15.8(14.5-17.3)$ & $66.7(54.8-76.8)$ \\
\hline $\begin{array}{l}\text { Negative predictive value } \\
(\%)\end{array}$ & $99.1(96.8-99.8)$ & $98.8(92.4-99.8)$ & $98.8(96.4-99.6)$ \\
\hline Positive likelihood ratio & $9.94(6.73-14.69)$ & $1.45(1.3-1.61)$ & $15.39(9.31-25.44)$ \\
\hline Negative likelihood ratio & $0.07(0.02-0.27)$ & $0.09(0.01-0.63)$ & $0.1(0.03-0.29)$ \\
\hline P value & $0.001^{*}$ & $0.001 *$ & $0.001 *$ \\
\hline
\end{tabular}

$* \mathrm{p}<0.05$ was accepted to be statistically significant.

\section{DISCUSSION}

Pap smear has been regarded as the most commonly used screening method for cervical cancer but its sensitivity is limited for the diagnosis of CIN3 or cervical cancer. On the other hand, pap smear is more able to detect CIN1 and CIN2. ${ }^{15}$ The identification of high risk HPV in the development of cervical cancer has led to the integration of HPV DNA testing into the screening programs for cervical cancer. Despite its high sensitivity, high risk HPV DNA testing usually fails to distinguish between frequently encountered transient infections and less prevalent premalignant lesions. ${ }^{15}$ These shortages of pap smear and HPV DNA tests end up with an increase in the number of referrals to colposcopy examination. ${ }^{15,16}$ Therefore, it has been hypothesized that biomarkers may help to differentiate between CINs that will regress and those that will persist so that individualized treatment of these precursor lesions would become possible. These biomarkers include viral factors, host factors and cellular factors such as p16, Ki-67, p53 and Rb. ${ }^{16,17}$

Dual staining for p16/Ki-67 in cervical cytology specimens has been addressed as a promising approach for further evaluation of patients with abnormal pap smear and/or HPV DNA test results. ${ }^{18}$ Most of the related studies report high sensitivity and sensitivity of p16/Ki-67 dualstaining for CIN3/cervical cancer. ${ }^{18-22}$ Ordi et al also specified that sensitivity and sensitivity of p16/Ki-67 dualstaining remained high in women aged less than 30 years and women aged older than 30 years. ${ }^{23}$
Apart from literature, this study aimed to investigate the efficiency of p16 and Ki-67 immunostaining for the detection of CIN lesions in a low risk population. Similar to a high risk population with abnormal screening results, positive staining for p16 in cervical cytology specimens had a sensitivity of $78.2 \%$ and a specificity of $97.4 \%$ and positive staining for Ki-67 in cervical cytology specimens had a sensitivity of $80.6 \%$ and a specificity of $57.9 \%$ for distinguishing CIN lesions in this study. Moreover, concurrent positive staining for p16 and Ki-67 in cervical cytology specimens had a sensitivity of $80.6 \%$ and a specificity of $97.4 \%$.

Wang et al were the first to investigate the diagnostic accuracy of p16 staining for differential diagnosis of CIN lesions. The sensitivity, specificity, positive and negative predictive values were $100 \%, 95 \%, 13.9 \%$ and $100 \%$ for the detection of CIN3 by p16 immunostaining. ${ }^{24}$ Positive p16 staining was documented in $0 \%$ to $15 \%$ of benign cervical lesions, $10 \%$ to $25 \%$ of CIN $1,45 \%$ to $100 \%$ of CIN2/3 and $80 \%$ to $100 \%$ of cervical cancers. ${ }^{25,26}$ The sensitivity and specificity of p16 staining were $91.3 \%$ and $98.1 \%$ for distinguishing CIN from non-dysplastic cervical lesions. $^{27}$

The over-expression of Ki-67 was directly associated with the severity of dysplasia. ${ }^{28}$ A Thai study observed Ki-67 expression in $11.3 \%$ of benign cervical lesions, $22.6 \%$ of CIN $1,75 \%$ of CIN2/3 and $100 \%$ of all invasive carcinomas. ${ }^{26}$ This finding complied with the findings of prior studies which found $\mathrm{Ki}-67$ in $0 \%$ to $20 \%$ of nondysplastic lesions, $70 \%$ to $90 \%$ of CIN1, $20 \%$ to $70 \%$ of CIN2/3 and $90 \%$ to $100 \%$ of invasive carcinomas..$^{29,30}$ The 
sensitivity and specificity of positive Ki-67 staining were $95.6 \%$ and $85.1 \%$ respectively for differentiating CIN from non-dysplastic cervical lesions. ${ }^{27}$

As for the $\mathrm{p} 16 / \mathrm{Ki}-67$ positivity, it correlated directly with the severity of cervical dysplasia, from $26.8 \%$ in normal histology, $46.5 \%$ in CIN1, $82.8 \%$ in CIN2 to $92.8 \%$ in CIN3. ${ }^{22}$ The sensitivity of $\mathrm{p} 16 / \mathrm{Ki}-67$ positivity altered between $93.2 \%$ and $100 \%$ and it specificity ranged from $46.1 \%$ to $74.2 \%$ for the detection of CIN3/cervical cancer. ${ }^{31}$ In case of detecting $\geq \mathrm{CIN} 3$ lesions, the sensitivity of p16/Ki67 positivity was statistically similar to that of high risk HPV DNA positivity but its specificity was significantly higher. ${ }^{22,31}$

In this study, positive staining for p16 in colposcopic biopsy specimens had a sensitivity of $94 \%$ and a specificity of $90.6 \%$ whereas positive staining for $\mathrm{Ki}-67$ in colposcopic biopsy specimens had a sensitivity of $97 \%$ and a specificity of $33 \%$ for differentiating CIN lesions. Positive staining for both p16 and Ki-67 in colposcopic biopsy specimens was found to have a sensitivity of $91 \%$ and a specificity of $94 \%$ for CIN lesions.

The findings of the present study should be interpreted carefully as their power was limited by the relatively small study cohort and the lack of dual immunostaining. Another limitation was the relatively high number of patients with CIN1 and, thus, the relatively low number of patients with CIN3.

\section{CONCLUSION}

It has been well established that the success of cervical cancer screening programs might be improved by biomarkers that specifically reflect the pathogenesis of HPV infections. These biomarkers usually aim to designate the expression of E6 and E7 oncogenes in basal keratinocytes affected by HPV. Theoretically, p16 and Ki67 typically induce opposite effects and the co-expression of p16 and Ki-67 protein would not occur physiologically. If the co-expression of p16 and Ki-67 would be detected, this would indicate the presence of HPV-related alterations and the need for more accurate histopathological examination. An assay of p16/Ki-67 immunohistochemistry applied on cervical cytology specimens could be a valuable screening test with high sensitivity and specificity for the detection of CIN2 and CIN3 lesions in high risk population. Further research is warranted to clarify the efficiency of p16/Ki-67 immunostaining in screening pre-invasive cervical lesions.

Funding: No funding sources Conflict of interest: None declared

Ethical approval: The study was approved by the Institutional Ethics Committee

\section{REFERENCES}

1. Bray F, Ferlay J, Soerjomataram I, Siegel RL, Torre LA, Jemal A. Global cancer statistics 2018: GLOBOCAN estimates of incidence and mortality worldwide for 36 cancers in 185 countries. CA Cancer J Clin. 2018;68(6):394-424.

2. Schiffman M, Castle PE, Jeronimo J, Rodriguez AC, Wacholder S. Human papillomavirus and cervical cancer. Lancet. 2007;370(9590):890-907.

3. Stiasny A, Kuhn C, Mayr D, Alexiou C, Janko C, Wiest I, et al. Immunohistochemical evaluation of E6/E7 HPV oncoproteins staining in cervical cancer. Anticancer Res. 2016;36(6):3195-8.

4. Hu D, Zhou J, Wang F, Shi H, Li Y, Li B. HPV-16 E6/E7 promotes cell migration and invasion in cervical cancer via regulating cadherin switch in vitro and in vivo. Arch Gynecol Obstet. 2015;292(6):134554.

5. Curry SJ, Krist AH, Owens DK, Barry MJ, Caughey $\mathrm{AB}$, Davidson $\mathrm{KW}$, et al. Screening for cervical cancer: US preventive services task force recommendation statement. JAMA. 2018;320(7):67486.

6. Saslow D, Solomon D, Lawson HW, Killackey M, Kulasingam SL, Cain J, et al. American cancer society, American society for colposcopy and cervical pathology, and American society for clinical pathology screening guidelines for the prevention and early detection of cervical cancer. Am J Clin Pathol. 2012;137(4):516-42.

7. Wang W, Zhang XH, Li M, Hao CH, Zhao ZM, Liang HP. Association between viral loads of different oncogenic human papillomavirus types and the degree of cervical lesions in the progression of cervical Cancer. Clin Chim Acta. 2018;483:249-55.

8. Lambert AF, Anshau F, Schmitt. P16 expression in cervical premalignant and malignant lesions. Exp Mol Pathol. 2006;80(2):192-6.

9. Mills AM, Dirks DC, Poulter MD, Mills SE, Stoler MH. HR-HPV E6/E7 mRNA in situ hybridization: validation against PCR, DNA in situ hybridization, and p16 immunohistochemistry in 102 samples of cervical, vulvar, anal, and head and neck neoplasia. Am J Surg Pathol. 2017;41(5):607-15.

10. Brown CA, Bogers J, Sahebali S, Depuydt CE, Prins F, Malinowski DP. Role of protein biomarkers in the detection of high-grade disease in cervical cancer screening programs. J Oncol. 2012;2012:289315.

11. Iaconis L, Hyjek E, Ellenson LH, Pirog EC. p16 and Ki-67 immunostaining in atypical immature squamous metaplasia of the uterine cervix: correlation with human papillomavirus detection. Arch Pathol Lab Med. 2007;131(9):1343-9.

12. Nam EJ, Kim JW, Hong JW, Jang HS, Lee SY, Jang SY, et al. Expression of the p16 and Ki-67 in relation to the grade of cervical intraepithelial neoplasia and high-risk human papillomavirus infection. J Gynecol Oncol. 2008;19(3):162-8. 
13. Solomon D, Davey D, Kurman R, Moriarty A, O'Connor D, Prey M, et al. The 2001 Bethesda System: terminology for reporting results of cervical cytology. JAMA. 2002;287(16):2114-9.

14. Wentzensen N, Schwartz L, Zuna RE, Smith K, Mathews C, Gold MA, et al. Performance of p16/Ki67 immunostaining to detect cervical cancer precursors in a colposcopy referral population. Clin Cancer Res. 2012;18(15):4154-62.

15. Areán-Cuns C, Mercado-Gutiérrez M, PanielloAlastruey I, Mallor-Giménez F, CórdobaIturriagagoitia A, Lozano-Escario $\mathrm{M}$, et al. Dual staining for $\mathrm{p} 16 / \mathrm{Ki} 67$ is a more specific test than cytology for triage of HPV-positive women. Virchows Arch. 2018;473(5):599-606.

16. Schiffman M, Wentzensen N. From human papillomavirus to cervical cancer. Obstet Gynecol. 2010;116(1):177-85.

17. Koeneman MM, Kruitwagen RF, Nijman HW, Slangen BF, Gorp TV, Kruse AJ. Natural history of high-grade cervical intraepithelial neoplasia: a review of prognostic biomarkers. Expert Rev Mol Diagn. 2015;15(4):527-46.

18. Schmidt D, Bergeron C, Denton KJ, Ridder R, European CINtec Cytology Study Group. p16/ki-67 dual-stain cytology in the triage of ASCUS and LSIL papanicolaou cytology: results from the European equivocal or mildly abnormal Papanicolaou cytology study. Cancer Cytopathol. 2011;119(3):158-66.

19. Waldstrom M, Christensen RK, Ornskov D. Evaluation of p16(INK4a)/Ki-67 dual stain in comparison with an mRNA human papillomavirus test on liquid-based cytology samples with low-grade squamous intraepithelial lesion. Cancer Cytopathol. 2013;121(3):136-45.

20. Loghavi S, Walts AE, Bose S. CINtec(R) plus dual immunostain: a triage tool for cervical pap smears with atypical squamous cells of undetermined significance and low grade squamous intraepithelial lesion. Diagn Cytopathol. 2013;41(7):582-7.

21. Petry KU, Schmidt D, Scherbring S, Luyten A, Reinecke-Lüthge A, Bergeron C, et al. Triaging Pap cytology negative, HPV positive cervical cancer screening results with p16/Ki67 dual-stained cytology. Gynecol Oncol. 2011;121(3):505-9.

22. Sahasrabuddhe VV, Luhn P, Wentzensen N. Human papillomavirus and cervical cancer: biomarkers for improved prevention efforts. Future Microbiol. 2011;6(9):1083-98.

23. Ordi J, Sagasta A, Munmany M, RodriguezCarunchio L, Torné A, Pino MD. Usefulness of p16/Ki67 immunostaining in the triage of women referred to colposcopy. Cancer Cytopathol. 2014;122(3):227-35.

24. Wang SS, Trunk M, Schiffman M, Herrero R, Sherman ME, Burk RD, et al. Validation of p16INK4a as a marker of oncogenic human papilloma virus infection in cervical biopsies from a population-based cohort in Costa Rica. Cancer Epidemiol Biomarkers Prev. 2004;13(8):1355-60.

25. Xing Y, Wang C, Wu J. Expression of geminin, p16, and Ki67 in cervical intraepithelial neoplasm and normal tissues. Medicine (Baltimore). 2017;96(26):7302.

26. Kanthiya K, Khunnarong J, Tangjitgamol S, Puripat $\mathrm{N}$, Tanvanich S. Expression of the p16 and Ki67 in cervical squamous intraepithelial lesions and cancer. Asian Pac J Cancer Prev. 2016;17(7):3201-6.

27. Aslani FS, Safaei A, Pourjabali M, Momtahan M. Evaluation of Ki67, p16 and CK17 markers in differentiating cervical intraepithelial neoplasia and benign lesions. Iran J Med Sci. 2013;38(1):15-21.

28. Conesa-Zamora P, Doménech-Peris A, OrantesCasado FJ, Ortiz-Reina S, Sahuquillo-Frías L, AcostaOrtega J, et al. Effect of human papillomavirus on cell cycle-related proteins p16, Ki-67, Cyclin D1, p53, and ProEx $\mathrm{C}$ in precursor lesions of cervical carcinoma: a tissue microarray study. Am J Clin Pathol. 2009;132(3):378-90.

29. Cavalcante DM, Linhares LM, Pompeu ML, Giraldo PC, Eleuterio J. The utility of p 16INK4a and Ki-67 to identify hig-grade squamous intraepithelial lesion in adolescents and young women. Indian $\mathrm{J}$ Med Microbiol. 2012;55(3):339-42.

30. Jackson JA, Kapur U, Ersahin C. Utility of p16, Ki67, and HPV test in diagnosis of cervical intraepithelial neoplasia and atrophy in women older than 50 years with 3- to 7-year follow-up. Int J Surg Pathol. 2012;20(2):146-53.

31. Zhu Y, Ren C, Yang L, Zhang X, Liu L, Wang Z. Performance of p16/Ki67 immunostaining, HPV E6/E7 mRNA testing, and HPV DNA assay to detect high-grade cervical dysplasia in women with ASCUS. BMC Cancer. 2019;19(1):271.

Cite this article as: Sahin B, Arioz DT, KanatPektas M, Ozdemir C, Cura G. Efficiency of p16 and Ki-67 immunostaining for detecting premalignant cervical lesions in high risk population. Int J Reprod Contracept Obstet Gynecol 2021;10:2578-83. 